\title{
How China should do to control the cancer well
}

\begin{abstract}
Background: In order to control the cancer, this article provided the facts how China should do to copy with uncontrolled cancers diseases in China.

Study Design: Comparison can find the gap. When the gap has been found. We can easily find solution. While China cancer control has been being in failing. But the cancer control in America has been better and better. So the administration gap of cancer control between China and the United States can be found. One of the most important things on administration of cancer control is how to do the cancer statistics. So comparing with international standard and Cancer statistics in America, we can find the shortcomings in China. Managing to change the shortcomings into rights. How China should do to control the cancer well could be found.
\end{abstract}

Methods: Through deeply analyzing the article about the latest cancer statistics in China, 2015, comparing with international standard and Cancer statistics in America, the shortcomings have been found.

Results: The shortcomings about cancer statistics in China have been found. The strategies for promotion and enhancement of the Chinese cancer statistics have been suggested as those. All provinces in China, covering 100\% Chinese, must build at least one standard representative sample of Contributing Cancer Registries. The former low quality of Population-Based Cancer Registries in China used for Incidence/Mortality Estimates, Temporal Trends, or Survival Estimates must be reformed to standard World Health Organization standard, and others. The quality of cancer epidemiology research in cancer statistics in America is much too higher than the quality of cancer epidemiology research in cancer statistics in China. That is why the China cancer control is much too less effective than the America's.

Conclusions: The Cancer statistics in America can be referenced as a model to do Cancer statistics in China and other countries. The low quality of cancer statistics in China and its bad cancer control has provided the valuable experiences for China and other countries to do our cancer statistics well and so as to control the cancer well. So as the cancer control all over the world could be as effective as Americans.

Keywords: cancer statistics, public health, cancer control, health management.
Volume 7 Issue 6 - 2019

\section{Xu Han-You}

Department of High Dependency Unit, Tongxiong Oriental Hospital, China

Correspondence: Xu Han-You, Department of High Dependency Unit, Tongxiong Oriental Hospital, Zhejiang Province, China, Tel 0086-|55|8962531,

Email abc|3579-you@|26.com

Received: October 27, 2019 | Published: November 18, 2019

\section{Introduction}

Cancer is a major public health problem worldwide. As the cancer is still a heavy burden on societies and people. And there have been not well control method in most of countries in the world. The cancer statistics are important to provide the scientific policies to control the cancer. As we have known. In America, the continuous decline in cancer death rates over 2 decades has resulted in an overall drop of $23 \%$, resulting in more than 1.7 million cancer deaths averted. ${ }^{1}$ But in China, the cancer death rates and morbidity have been continuing with uncontrolled. Though the cancer control is a complex project. Which includes, but not limited, the development of cancer research science, national social development, national economic strength, administration of the public health promotion, national civilization and education, etc.. Among the factors causing the bad cancer control in China, one of the important factor is that cancer statistics in China is bad and not scientific. In this article, comparing the cancer statistics in America, we can find where the cancer statistics in China is not scientific and which important factors have caused the bad cancer control in China. So as to enhance the cancer control in China.

\section{Method and study design}

It is well known that comparison can find the gap. When the gap has been found. We can easily find solution. While China cancer control has been being in failing. But the cancer control in America has been better and better. So the administration gap of cancer control between China and the United States can be found. One of the most important things on administration of cancer control is how to do the cancer statistics. So comparing with international standard and Cancer statistics in America, we can find the shortcomings in China. Managing to change the shortcomings into rights. How China should do to control the cancer well could be found. Through deeply analysing the article about the latest cancer statistics in China, comparing with international standard and Cancer statistics in America, the shortcomings and the gaps have been found.

\section{The basic collection of incidence and mortality data in America}

From 1930 to 2012, mortality data were provided by the National Center for Health Statistics. ${ }^{2,3}$ There are 47 states and the District of 
Columbia met data quality requirements for reporting to the national vital statistics system in 1930. But Texas began reporting mortality data in 1933. While Alaska, and Hawaii began reporting mortality data in 1959 and 1960, respectively.

Up to now, the incidence data has approached $100 \%$ coverage of the US population in the most recent time period and were the source for the projected new cancer cases in 2016 and incidence rates by state and race/ethnicity. ${ }^{1}$

All cancer cases were classified according to the International Classification of Diseases for Oncology. But the childhood and adolescent cancers were classified according to the International Classification of Childhood Cancer .Causes of death were classified according to the International Classification of Diseases.All incidence and death rates were age-standardized to the 2000 US standard population and expressed per 100,000 population, as calculated by National Cancer Institute's Surveillance, Epidemiology, and End Results (SEER)*Stat software (version 8.2.1). The annual percent change in rates was quantified using National Cancer Institute's Joinpoint Regression Program (version 4.2.0.2).

\section{The basic collection of incidence and mortality data in China}

According to the latest cancer statistics in China, published by CA: A Cancer Journal for Clinicians by Wanqing Chen et al., ${ }^{4}$ we can find easily the following shortcomings, but not limited.

\section{Results}

\section{The cancer incidence data included invasive tumors only in this study is wrong}

The World Health Organization (WHO) has guarded the International Classification of Diseases (ICD). ${ }^{5}$ Which the ICD is the united international standard diagnostic tool for epidemiology, health management and clinical purposes, including, but not limited, the analysis of the general health situation of population groups. It is also used to monitor the incidence and prevalence of diseases and other health problems, proving a picture of the general health situation of countries and populations. In addition to enabling the storage and retrieval of diagnostic information for clinical, epidemiological and quality purposes, these records also provide the basis for the compilation of national mortality and morbidity statistics by WHO Member States. So as to control the diseases and health problems around the world.

The ICD-10 was endorsed by the Forty-third World Health Assembly in May 1990 and came into use in WHO Member States as from 1994. ICD-10 is currently widely used by WHO Member States, including China. While ICD-O-3 is International Classification of Diseases for Oncology, 3rd Edition. ${ }^{6}$ No matter ICD-10 or ICD-O-3, the cancer classification all has malignant, benign, or uncertain (whether benign or malignant). But classification of ICD-O-3 includes malignant, benign, in situ, or uncertain (whether benign or malignant). In China, all the hospitals have been using the ICD-10 classification governed by National Health and Family Planing Commission. So the cancer classification includes malignant, benign, or uncertain (whether benign or malignant). In this article of latest cancer statistics in China, ${ }^{4}$ they clearly stated that incidence data and mortality data all using the ICD-10 classification. Therefore, their classification only include malignant, benign, or uncertain (whether benign or malignant).
In oncology, the basic knowledge is that malignant tumors include invasive malignant tumors, before the stage of invasive malignant tumors and primary malignant tumors or in situ. So the invasive malignant tumors, before the stage of invasive malignant tumors and primary malignant tumors are all included into the ICD10 classification. But the latest cancer statistics in China, ${ }^{4}$ the cancer incidence data included invasive tumors only in this study. So their study for cancer incidence data should be more scientific and abide by international standard for ICD-10 classification. And their numbers of cancer incidence data are less than the real numbers of standard ICD-10 classification. Therefore, the study results and conclusion data of the cancer statistics are much too less accurate and precise. So the cancer incidence data included invasive tumors only in their study is only created wrongly by its authors without international standard.

\section{The mortality data collection is much too small than the real data}

While the article of the Chinese latest cancer statistics published by CA: A Cancer Journal for Clinicians by Chen et al., ${ }^{4}$ said the mortality data compiled by 72 local cancer registries were used to estimate the numbers of cancer deaths in China in 2015. To estimate trends in cancer mortality between 2000 and 2011, mortality data were obtained from the same 22 local registries that were used in the incidence trend analyses. These registries compile data on cancer deaths from local hospitals, community health centers, vital statistics (including data from the national Disease Surveillance Points [DSP] system), and the Civil Administration Bureau. The DSP system, which was established by the Ministry of Health in the early 1980s, routinely collects information on deaths based on the death certificate provided by hospitals or obtained from the next of kin by a household visit if a death certificate was unavailable. While the DSP uses a nationally representative sample of sites, these cover only a very small $(\sim 1 \%)$ proportion of the population.

In fact, the real situation in China as a first hand encountering by the author is that, in Chinese habit and real situation, lots of sources of cancer mortality data have not be included into the research with above mentioned sources. For example, the cancer patients as peasants have been dying at ten millions of patients at township hospitals, even at county hospitals and at their own home in countryside of China have not registered any at above mentioned sources for this research. For the cancer patients as peasants, they usually are cared at local hospitals when their conditions are hopeful to recovery. But when their conditions are critical with no hope to recovery. The families of cancer patients will take them back to homes waiting to death before they die at hospitals. So the hospitals record the cancer patients are not dead. The peasants usually do not need death certificates. While according to the article, the local cancer incidence and death registries as representative sample of sites, have been covering only a very small $(\sim 1 \%)$ proportion of the population. So the included data is much too small than the real mortality data.

\section{The contributing cancer registries and geographic regions in china are much too less balanced}

From the map for 22 cancer registries (data from 2000 to 2011)and the map for 72 cancer registries (data from 2009 to 2011), we can see clearly that the Contributing Cancer Registries are mainly concentrated at central China, East China, South China and Southeast China. While North China, Northeast China, Northwest China, Southwest China and West China have much too less or no Contributing Cancer 
Registries. Where the Geographic Regions of less or no Contributing Cancer Registries usually are less developed and may be more cancer incidence and mortality.

The quality of population-based cancer registries in China used for incidence/mortality estimates, temporal trends, or survival estimates in cancer statistics in China is not up to the WHO standard judged by the International Agency for Research on Cancer (IARC)

In the article, the related table has the list of Population-Based Cancer Registries in China used for Incidence/Mortality Estimates, Temporal Trends, or Survival Estimates. Only these labeled "a" are registries from which data were accepted by IARC for the most recent publication of Cancer Incidence in Five Continents (2014). For all the 73 Population-Based Cancer Registries, only 11 Population-Based Cancer Registries have been accepted and used by IARC for the most recent publication of Cancer Incidence in Five Continents (2014). Which has been expressing that data quality is low or at least under question. Because for epidemiology, health management and clinical purposes by WHO to publish the qualify Cancer Incidence in Five Continents, the more Population-Based Cancer Registries are, the better the quality is. While more than 70 Population-Based Cancer Registries in China, why IARC chose only 11 Registries? The main reason is that the other Population-Based Cancer Registries in China is low quality and no valuable to be included into the data collection.

\section{Cancer statistics in America, 2016 is the example of} cancer epidemiology research for cancer control

The cancer statistics in America, $2016^{1}$ published at the same journal of CA: A Cancer Journal for Clinicians has provided high quality of cancer epidemiology research. In the research, all states of America have standard representative sample of sites for PopulationBased Cancer Registries and strictly accepted the standard of ICD-O-3 classification for the statistics in a long time. This has paved the way for the United States of America to control the cancer well. So it is easy to compare that the quality of cancer epidemiology research in Cancer statistics in America, 2016 is much too higher than the quality of cancer epidemiology research in the latest Cancer statistics in China. That is why the China cancer control is much too less effective than the America's.

\section{Discussion}

While the cancer control in China has been observing the Chinese suffering from uncontrolled cancers diseases. We can easily find the lots of shortcomings at the latest and the best cancer statistics in China, published by CA: A Cancer Journal for Clinicians by Wanqing Chen et al. ${ }^{4}$ And when the shortcomings at the latest and the best cancer statistics in China could be corrected easily, but not corrected. So it is the time to change imperatively. It is basic inclusion criteria for high quality research of cancer epidemiology that the data quality must be right, correct, accurate and precise. Which include that the inclusion criteria must be confirmed to the international standard.

As for Cancer statistics in China, the basic inclusion criteria for the qualify data is the international standard of ICD-10 classification. But the data are far from the international standard of ICD-10 classification by WHO. The cancer incidence data included invasive tumors only in the latest cancer statistics in China. The cancer mortality data has not been fully collected. The Contributing Cancer Registries and Geographic Regions in China are much too less balanced. The quality of Population-Based Cancer Registries in China used for Incidence/ Mortality Estimates, Temporal Trends, or Survival Estimates is not up to the WHO standard judged by IARC. Which at least four important aspects of data inclusion were not correct and accurate. So the results of the latest cancer statistics in China should be more correct and accurate.

Of course, up to now, any cancer research or medical research has not been shortcoming free. But the shortcomings at the cancer research must be as faint as possible. Which reaches to such a deep and grade that the researchers cannot reduce any shortcomings further according to modern international standard and natural limitations. But the latest cancer statistics in China, at least has four important aspects of data inclusion should be more correct and accurate. But these shortcomings in the four important aspects of data inclusion can be easily promoted and enhanced to modern international standard. It is well known that China cancer control is bad. Comparing the cancer statistics in America, the quality of cancer epidemiology research in Cancer statistics in America is much too higher than the quality of cancer epidemiology research in Cancer statistics in China. That is why the China cancer control is much too less effective than the America's.

So at this occasion, I suggest that Chinese cancer incidence and mortality data for cancer epidemiology study must include all malignant tumors in ICD-10 classification or ICD-O-3 classification. The cancer mortality data must be fully collected by totally collecting all data in standard representative sample of sites. The Contributing Cancer Registries and Geographic Regions in China must be balanced. The North China, Northeast China, Northwest China, Southwest China and West China where at present have much too less or no Contributing Cancer Registries must build standard representative sample of Contributing Cancer Registries. The former low quality of Population-Based Cancer Registries in China used for Incidence/ Mortality Estimates, Temporal Trends, or Survival Estimates must be reformed to standard WHO standard. While the new PopulationBased Cancer Registries must build standard representative sample of sites at the beginning. So all provinces in China, covering $100 \%$ Chinese, must build at least one standard representative sample of Contributing Cancer Registries. The research of cancer statistics in China should like cancer statistics in America, $2016 .{ }^{1}$

\section{Conclusion}

After carefully analyzing the article of the latest Cancer statistics in China, comparing that with the America's, we can easily find that the research quality of Cancer statistics in China is much too low. This is one of main reason why China cancer control is bad. So the high quality of Cancer statistics in China is needed as soon as possible to guard the Chinese cancer control. This research provides the timely proposals to enhance the quality of Cancer statistics in China. The Cancer statistics in America can be referenced as a model to do Cancer statistics in China and other countries. The low quality of Cancer statistics in China and its bad cancer control has provided the valuable experiences for other countries to do their Cancer statistics well and so as to control their cancer well after this candid research are published and referenced by Chinese and international societies. 


\section{Acknowledgments}

None.

\section{Conflict of interest}

Author declares that there is no conflict of interest.

\section{References}

1. Siegel RL, Miller KD, Jemal A. Cancer statistics, 2016. CA Cancer J Clin. 2016;66(1):7-30.

2. Surveillance, Epidemiology, and End Results (SEER) Program. SEER*Stat Database: Mortality-All COD, Aggregated With State, Total US (1969-2012) < Katrina/Rita Population Adjustment>. Bethesda, MD: National Cancer Institute, Division of Cancer Control and Population Sciences, Surveillance Research Program, Cancer Statistics Branch; 2015 .
3. Wingo PA, Cardinez CJ, Landis SH, et al. Long-term trends in cancer mortality in the United States, 1930-1998. Cancer. 2003;97(Suppl 12):3133-3275.

4. Chen W, Zheng R, Baade PD, et al.. Cancer statistics in China, 2015. CA Cancer J Clin. 2016;66(2):115-132.

5. International Classification of Diseases (ICD). WHO; 2016.

6. International Classification of Diseases for Oncology, 3rd Ed. WHO; 2016. 